\section{BRAZIULIAN JOURNAL}

OF MEDICAL AND BIOLOGICAL RESFARCH

www.bjournal.com.br
ISSN 0100-879X

Volume 43 (7) 600-697 July 2010

BIOMEDICAL SCIENCES

AND

CLINICAL INVESTIGATION

Braz J Med Biol Res, July 2010, Volume 43(7) 600-610

doi: 10.1590/S0100-879X2010007500044

Autonomic control of cardiorespiratory interactions in fish, amphibians and reptiles

E.W. Taylor, C.A.C. Leite and N. Skovgaard

The Brazilian Journal of Medical and Biological Research is partially financed by
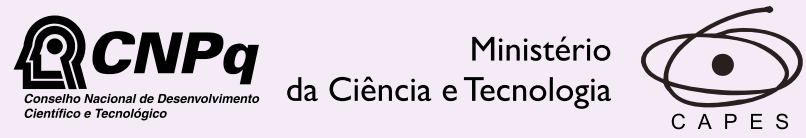

Ministério da Educação

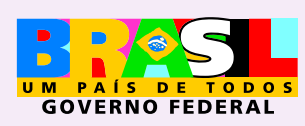

DFAPESP

Institutional Sponsors
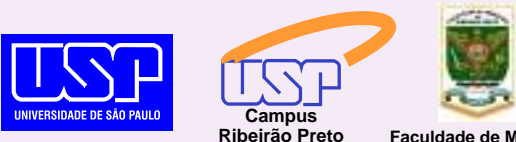

$\oplus$ SHIMADZU

GE Healthcare
Hotsite of proteomics metabolomics developped by: 


\title{
Autonomic control of cardiorespiratory interactions in fish, amphibians and reptiles
}

\author{
E.W. Taylor ${ }^{1,2,3}$, C.A.C. Leite ${ }^{2,3}$ and N. Skovgaard 4 \\ ${ }^{1}$ School of Biosciences, University of Birmingham, Birmingham, UK \\ 2Departamento de Zoologia, Instituto de Biociências, Universidade Estadual Paulista, Rio Claro, SP, Brasil \\ ${ }^{3}$ Instituto Nacional de Ciência e Tecnologia em Fisiologia Comparada, Rio Claro, SP, Brasil \\ ${ }^{4}$ Department of Pharmacology, University of Aarhus, Aarhus, Denmark
}

\begin{abstract}
Control of the heart rate and cardiorespiratory interactions (CRI) is predominantly parasympathetic in all jawed vertebrates, with the sympathetic nervous system having some influence in tetrapods. Respiratory sinus arrhythmia (RSA) has been described as a solely mammalian phenomenon but respiration-related beat-to-beat control of the heart has been described in fish and reptiles. Though they are both important, the relative roles of feed-forward central control and peripheral reflexes in generating CRI vary between groups of fishes and probably between other vertebrates. CRI may relate to two locations for the vagal preganglionic neurons (VPN) and in particular cardiac VPN in the brainstem. This has been described in representatives from all vertebrate groups, though the proportion in each location is variable. Air-breathing fishes, amphibians and reptiles breathe discontinuously and the onset of a bout of breathing is characteristically accompanied by an immediate increase in heart rate plus, in the latter two groups, a left-right shunting of blood through the pulmonary circuit. Both the increase in heart rate and opening of a sphincter on the pulmonary artery are due to withdrawal of vagal tone. An increase in heart rate following a meal in snakes is related to withdrawal of vagal tone plus a non-adrenergic-non-cholinergic effect that may be due to humoral factors released by the gut. Histamine is one candidate for this role.
\end{abstract}

Key words: Cardiorespiratory interactions; Autonomic nervous system; Cardiac vagal preganglionic neurons; Central interactions; Peripheral reflex control; Non-adrenergic-non-cholinergic (NANC) control of heart rate

\section{Introduction}

\section{The control of the heart}

The vertebrate heart beats in response to phasic activity in the cardiac pacemaker, which, in turn, operates under the influence of a number of intrinsic and extrinsic factors. The basis for the regulation of heart beat is essentially similar from mammals to fish. Both factors affecting cardiac output, frequency and stroke volume, are controlled by the autonomic nervous system (ANS). Excepting the cyclostomes, all vertebrates have an inhibitory cholinergic influence exerted by the parasympathetic nervous system via the vagus nerve. The sympathetic nervous system exerts a cardio-excitatory influence over the heart in all tetrapod vertebrates but not in cyclostomes, elasmobranches and some teleost fishes. Despite this dual autonomic innervation of the heart in tetrapods, parasympathetic inhibition plays the dominant role in the regulation of heart rate, including heart rate variability (HRV) and in particular cardiorespiratory interactions in all jawed vertebrates (1). Here we briefly review our current understanding of cardiorespiratory control on mammals before moving on to consider the diversity of systems present in fish, amphibians and reptiles.

\section{Central nervous organization}

In mammals, central control of the cardiorespiratory system is known to be complex and interactive. Direct feed-forward control operates from the brainstem and this is modulated by afferent inputs from areas in the midbrain and forebrain such as the hypothalamus, amygdala and cortex (2). Heart rate may vary with emotional state and/or in several routine behavioral responses such as the classic "fight or flight response". The amygdala receives projections from

Correspondence: E.W. Taylor, School of Biosciences, University of Birmingham, B15 2TT, Birmingham, UK. E-mail: e.w.taylor@bham.ac.uk Presented at the XXIV Annual Meeting of the Federação de Sociedades de Biologia Experimental, Águas de Lindóia, SP, Brazil, August 19-22, 2009.

Received February 24, 2010. Accepted April 27, 2010. Available online May 14, 2010. Published July 9, 2010. 
several nuclei involved in cardiovascular control such as the hypothalamus, parabrachial nuclei, nucleus of the solitary tract (NTS), and dorsal motor column of the vagus $(2,3)$. In addition, it is linked to the infralimbic and insular cortices. Consequently, it is a critical site for cardiovascular control and has the role of integrating the autonomic responses to emotional stimuli like fear (2). The hypothalamus is also a key area for the control of ANS function since it integrates information from somatic motor areas, emotional state and also humoral efferent activity (3). Hypothalamic nuclei have direct connections with ventrolateral areas in the brainstem related to parasympathetic control of the cardiovascular system plus the intermediolateral neurons of the cervical and thoracic medulla related to sympathetic control (2).

\section{Reflex control}

Stimulation of a variety of cardiorespiratory afferents evokes changes in both respiratory and cardiovascular outflows and, at least at the peripheral level, these have been fully reviewed (4). For example, stimulation of arterial chemoreceptors augments respiratory drive in mammals, in addition to altering heart rate and vascular resistance. Since the cardiovascular response is modified by respiration, the overall response evoked by chemoreceptor stimulation is complex. The primary response to stimulating chemoreceptors is a slowing of the heart, and this is always seen if respiration is controlled. However, if respiration is allowed to increase, then this may mask the bradycardia and lead to tachycardia. This has been well documented in previous reviews $(5,6)$. During breath-hold diving, apnea is evoked by stimulation of facial receptors innervated by trigeminal afferents. The breath-hold leads to a progressive hypoxic stimulation of the arterial chemoreceptors, which would be expected to stimulate breathing. However, the simultaneous stimulation of the facial receptors blocks this respiratory component of the chemoreceptor reflex whilst at the same time augmenting the cardiac component $(1,7)$.

The NTS is the main site for primary afferent synapses from visceral receptors in mammals. This area receives information from receptors initiating a series of cardioregulatory reflexes such as the arterial baroreceptors, pulmonary stretch receptors and carotid chemoreceptors (8). The NTS is also the route for communication between the sympathetic adrenal axis in the medulla and more rostral areas of the central nervous system (3). Despite some structural differences, there are afferent projections into the brainstem of fish that appear to perform similar functions and may be homologous to the NTS in mammals (1). In cats, for instance, the NTS projects to respiratory neurons in the pons and to the dorsal and ventral respiratory groups of the medulla (9). In the catfish the sensory "NTS" projects to an area equivalent to what would be the parabrachial nucleus of the respiratory neuron group in the mammalian pons that incorporates gustatory and visceral sensory nuclei (10). The primary visceral sensory areas form the dorsolateral column at the side of the IV ventricle. In this column sensory afferent fibers from the VII, IX and X cranial nerves form a rostrocaudal sequence (11). Central injection of glutamate in the teleostean fish Myoxocephalus scorpius confirmed that this neurotransmitter has an important role in those pathways, participating in several cardiorespiratory responses. This demonstrates the importance of glutamate as a transmitter in the NTS of mammals (12).

\section{Cardiorespiratory interactions}

In mammals, the effectiveness of certain cardiac reflexes is markedly modified by respiration. Brief stimuli applied to the arterial baroreceptors or chemoreceptors only evoke reductions in heart rate if they are applied during expiration, stimuli given during inspiration being less effective or totally ineffective. Since the preganglionic neurons themselves are under respiratory control, it might be predicted that any cardiac reflex, which is mediated by these neurons, would be modulated by respiration. In contrast, stimulation of receptors in the airways and cardiac $\mathrm{C}$-fiber receptors all evoke reflex excitation of cardiac vagal outflow, potentially resulting in a bradycardia, which is modified by respiratory drive (reviewed in Ref. 1). Thus, the arterial chemoreceptorevoked bradycardia is almost abolished by lung inflation and during inspiration, whilst the bradycardia evoked by stimulating the baroreceptors and cardiac C-fibers is reduced by $50-60 \%$. Surprisingly, the bradycardia evoked by stimulation of pulmonary C-fiber afferents was completely unaffected by respiration (13). One interpretation of these data is that the bradycardia evoked by one group of afferents may be mediated by a different group of cardiac vagal preganglionic neurons (CVPN) to those activated by the other afferents. In mammals the CVPN are located in the dorsal motor column of the vagus (DVN), in the nucleus ambiguus (NA) and in an intermediate zone between those groups, with the majority (up to $70 \%$ ) being located in the NA (1). In both cats and rats, CVPN located in the DVN are activated by stimulation of pulmonary C-fibers but are unaffected by the arterial baroreceptors or the respiratory cycle (14). The ongoing activity of these neurons is rather regular, in contrast to that of neurons located in the NA, which fire with respiratory- and cardiac-related rhythms.

As a result of these interactions in the brainstem, heart rate in mammals increases during inspiration, a phenomenon known as respiratory sinus arrhythmia (RSA). It is known to be driven by respiration-related fluctuations in the efferent, inhibitory supply to the heart via the cardiac vagus. This is generated centrally by an inhibitory input from inspiratory neurons in the respiratory group to CVPN outside of the DVN in the ventrolateral NA. The consequent gating of vagal outflow causes heart rate to rise during inspiration (15). Such variability has a functional role in improving pulmonary oxygen uptake as recently discussed 
by Hayano and Yasuma (16).

Thus, as previously demonstrated in the dogfish (see below), mammals appear to have two functionally separate groups of CVPN, which have either tonic or phasic firing patterns and may be topographically separated into groups in the DVN or the NA. This separation arises during embryological development as neurons, which form the NA, migrate ventrolaterally from a more dorsomedial position, possibly the equivalent of the DVN, in the fetal brainstem (17). Power spectral analysis of recordings of heart rate and breathing movements in human neonates revealed that RSA is a major contributor to HRV in healthy term (38-40 weeks gestation) newborn infants (18). Although RSA was detected in the near-term fetus (over 35 weeks), it was not discernible in the fetus prior to this gestational age or in early premature neonates at less than 30 weeks, appearing later in postnatal development at around 33 weeks (Figure 1). Thus, the contribution of RSA to HRV varies both with pre- and postnatal age, which may reflect a maturational development of the underlying mechanisms (1). This is likely to reflect myelination of the nerve fibers supplied from CVPN in the NA that will increase transmission rates, enabling beat-by-beat control of heart rate, rather than ventrolateral migration of CVPN at this late stage of development. However, the onset of air-breathing at metamorphosis in the axolotl was accompanied by ventrolateral relocation of a subpopulation of vagal preganglionic neurons (VPN, see below).

The "polyvagal theory" has suggested that the beat-tobeat control of heart rate that generates RSA is restricted to mammals, which have evolved myelinated vagal pathways that originate in the NA (19). This assertion has recently been contested by Grossman and Taylor (20) who pointed out that beat-to-beat control of the heart, necessary to generate cardiorespiratory synchrony, has been reported in resting dogfish (21) and that rattlesnakes show a respiratory component in their HRV that resembles mammalian RSA (22) and that both of these species have CVPN in two locations in the brainstem. In air-breathing fish, amphibians and reptiles that breathe discontinuously, heart rate increases immediately at the onset of periodic ventilatory events and it has been demonstrated that this results largely from withdrawal of vagal activity to the heart, most likely due to central interactions between motor neurons driving both systems (1). In amphibians and reptiles with undivided ventricles, intracardiac or vascular shunts can cause an increase in pulmonary blood flow, relative to systemic flow, during bouts of ventilation. Periods of increased activity can result in an increase in heart rate and a reduced resistance in the pulmonary circuit, due to relaxation of a sphincter on the pulmonary artery. Both changes are due primarily to withdrawal of efferent vagal activity $(23,24)$. Here we review the role of the autonomic nervous system in generating integrated cardioventilatory responses in fish, amphibians and reptiles that arise both from central interactions and from cardiorespiratory reflexes.

\section{Fish}

The matching of the flow rates of water and blood according to their relative capacities for oxygen over the counter-current exchanger at the gills of fish is essential for effective respiratory gas exchange. The pumping action of the heart generates a pulsatile flow of blood, which in fish is delivered directly down the ventral aorta to the afferent branchial vessels. Blood flow remains markedly pulsatile
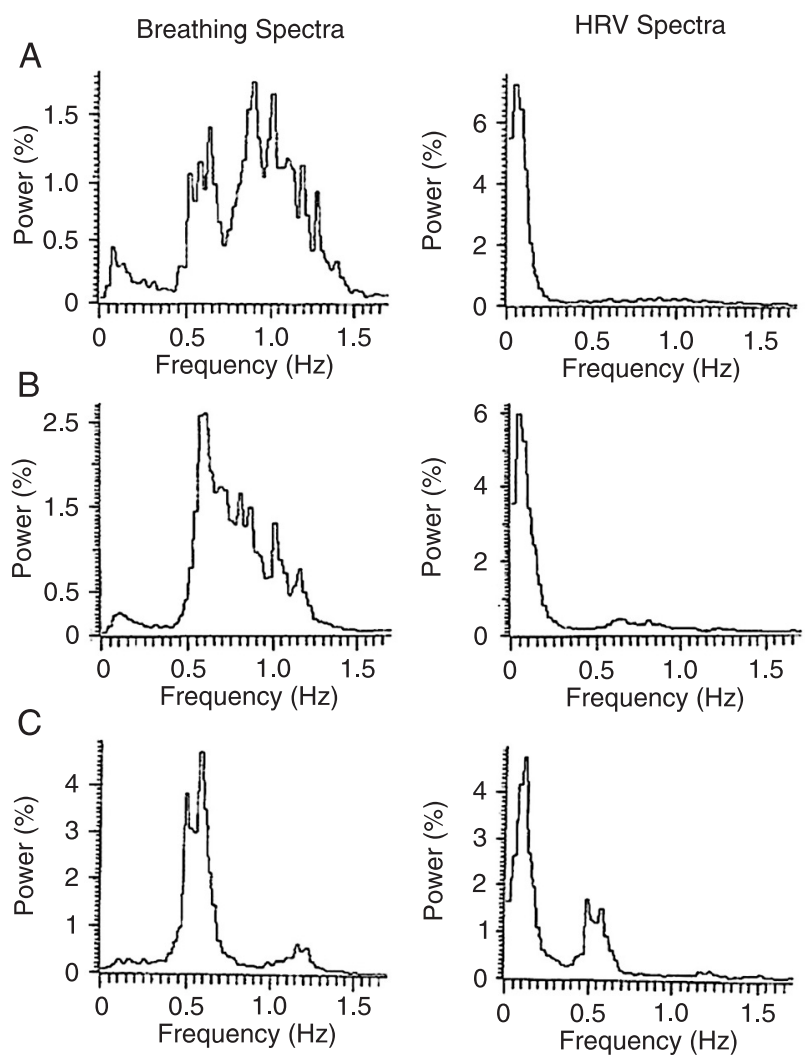

Figure 1. Power spectra of mean breathing and heart rate variability (HRV) from healthy premature neonates 2-5 days old having experienced different gestations: $A$, less than 30 weeks gestation $(N=5) ; B, 33$ weeks gestation $(N=12) ; C, 36$ weeks gestation $(\mathrm{N}=8)$. The spectra represent the relative distribution of frequency components within the HRV and breathing signals. There was no high frequency component, indicative of respiratory sinus arrhythmia in the HRV spectra from neonates born at less than 30 weeks gestation. Their breathing spectra cover a wide range of frequencies reflecting the arrhythmic breathing patterns of these neonates. A high frequency, respiration-related peak first appears in the HRV signal from neonates born at 33 weeks and is consolidated in those born at 36 weeks, when the neonates show a more stable range of breathing frequencies. A clear peak in HRV is seen (18) in neonates born at the normal term of 40 weeks (Thompson CR, Brown J, Gee H, Taylor EW, unpublished data). 
across the resistance offered by the branchial circulation. Water flow across the gill lamellae is also markedly pulsatile. Accordingly, some degree of synchronization of the two flows would seem likely to increase the effectiveness of respiratory gas exchange $(1,21)$. The improvement in gill perfusion and consequent oxygen transfer resulting from pulsatile changes in transmural pressure and intralamellar blood flow (25) may be further improved by synchronization of the pressure pulses associated with ventilation and perfusion. Cardiorespiratory synchrony may, by a combination of these effects, increase the relative efficiency of respiratory gas exchange (i.e., maximum exchange for minimum work).

Cardiorespiratory coupling (CRC) has been reported in resting dogfish and cod (21), hypoxic trout (26) and pacu $(27,28)$. Cardiac vagotomy or injection of atropine abolished cardiorespiratory synchrony in the dogfish (21), while in the sculpin, Myoxocephalus scorpius, injection of atropine raised mean heart rate in normoxia and abolished a hypoxic bradycardia while cardiac vagotomy abolished HRV (29). These observations confirm the dependence of beat-to-beat variability of heart rate in fish on tonic vagal control. Thus, we have established a potential functional role for $\mathrm{CRC}$ in optimizing respiratory gas exchange in fish and can attribute its generation to efferent vagal control of the heart. Failure to demonstrate CRC in fish can often be attributed to the highly invasive experimental techniques employed by some laboratories. What follows is a detailed account of the nature of vagal control of the heart in fish.

\section{Elasmobranches}

The heart in elasmobranch fishes such as the dogfish, Scyliorhinus canicula operates under an inhibitory parasympathetic tonus, in the absence of a sympathetic supply to the heart (21). Recordings from the central cut end of a cardiac branch of the vagus in decerebrated paralyzed dogfish revealed high levels of spontaneous efferent activity that could be attributed to two types of unit. Some units fired sporadically and increased their firing rate during hypoxia. Other, typically larger, units fired in rhythmical bursts, which were synchronous with ventilatory movements $(1,30)$. We hypothesized that these units, showing respiration-related activity, which was unaffected by hypoxia, may serve to synchronize heart beat with ventilation (21). This separation of efferent cardiac vagal activity into respiration-related and non-respiration-related units was discovered to have a basis in the distribution of their neuron cell bodies in the brainstem (31).

Extracellular recordings from CVPN identified in the hindbrain of dogfish by antidromic stimulation of a cardiac branch of the vagus revealed that neurons located in the DVN were spontaneously active, firing in rhythmical bursts, which contributed to the respiration-related bursts recorded from the intact nerve (32). Direct connections between bursting CVPN and rostral ventromedial medulla (RVM) are possible in the dogfish hindbrain, as both are located in the DVN with an overlapping rostrocaudal distribution. As the bursts are synchronous, the innervation of CVPN is likely to be excitatory rather than inhibitory as described for the mammal and it is equally possible that a direct drive from a central pattern generator operates both on the RVM and the CVPN (21). Neurons located ventrolaterally outside of the DVN were either spontaneously active, firing regularly or sporadically but never rhythmically, or were silent. Thus, the two types of efferent activity recorded from the cardiac nerve arise from the separate groups of CVPN, as identified by neuroanatomical studies (21).

As activity was recorded from the central cut end of the cardiac vagus, or centrally from CVPN, in the decerebrated paralyzed dogfish, provided with a constant, non-pulsatile supply of aerated water, the respiration-related activity recorded from CVPN in the DVN must have been generated by central interactions. However, all of the spontaneously active CVPN from both divisions and some of the silent CVPN fired in response to mechanical stimulation of a gill arch (32), indicating that stimulation of mechanoreceptors on the gills may recruit CVPN in normally breathing fish. Also, phasic electrical stimulation of the central cut end of a branchial branch of the vagus in the decerebrated dogfish entrained the efferent bursting units recorded from the central cut end of the ipsilateral branchial cardiac branch (21). Although this effect is likely to be attributable to stimulation of mechanoreceptor afferents it is not possible to eliminate the possibility that antidromic stimulation of branchial efferent fibers may have activated the RVM that then recruited CVPN by direct interactions in the DVN, where they have an overlapping distribution (1).

Thus, in the intact fish, normal breathing movements that stimulate peripheral mechanoreceptors on the gills may, by a reflex pathway, generate activity in CVPN and consequently in the cardiac vagi, affecting heart rate. This implies that the typical reflex bradycardia in response to hypoxia may arise both directly, following stimulation of peripheral chemoreceptors and indirectly, via increased ventilation, which by stimulating branchial mechanoreceptors may increase vagal outflow to the heart. This is reminiscent of, but opposite in kind to, the hypoxic response in the mammal, where stimulation of lung stretch receptors causes an increase in heart rate (33).

As well as slowing the heart, respiration-related efferent activity in the cardiac vagi may entrain the heart. This possibility was confirmed in this group by peripheral electrical stimulation of these nerves in the prepared dogfish. Although continuous vagal stimulation normally slowed the heart, it proved possible to drive the denervated heart at a rate either lower or somewhat higher than its intrinsic rate with brief bursts of stimuli, delivered down one branchial cardiac vagal branch (34). When bursts of electrical stimuli were delivered at 43 bursts/min in a preparation with an intrinsic heart rate of 36 beats/min the heart slowed but 
it was observed to beat at exactly half the bursting rate, implying that it was induced to beat by alternate bursts (34) rather than being simply inhibited.

Similar effects of peripheral stimulation of the cardiac vagus have been reported for mammals. In anesthetized dogs and cats electrical stimulation of the vagus nerve towards the heart with brief bursts of stimuli, similar to those recorded from efferent cardiac vagal fibers, caused heart rate to synchronize with the stimulus over a wide range of frequencies both lower and higher than the intrinsic heart rate (reviewed in Ref. 35). The mechanisms of beat-tobeat vagal control of heart rate in mammals remain largely unresolved but there are several studies that indicate that the effect of a brief burst of efferent activity delivered down the cardiac vagus relates to the phase of the cardiac cycle when it is delivered $(36,37)$. Thus, the vagal effect on the heart cannot be measured merely in terms of the amount of acetylcholine delivered per unit time. Similar detailed studies are long overdue on fish, amphibians and reptiles.

\section{Teleosts}

Work on teleosts has stressed the importance of inputs from peripheral receptors in the genesis of cardiorespiratory synchrony. Randall (26) recorded efferent nervous activity from the cardiac branch of the vagus in the tench that was synchronized with the mouth-opening phase of the breathing cycle. It was suggested that this activity generates synchrony between heart beat and breathing movements and that both a hypoxic bradycardia and synchrony were mediated by reflex pathways. Randall and Smith (38) described the development of an exact synchrony between breathing and heart beat in the trout during progressive hypoxia. In normoxia, heart rate was faster than ventilation; hypoxia caused an increase in ventilation rate and a reflex bradycardia, which converged to produce a 1:1 synchronization of the two rhythms. Both the bradycardia and synchrony were abolished by atropine. In addition, Randall and Smith (38) were able to demonstrate $1: 1$ synchronization of hypoxic heart rate with pulsatile forced ventilation, which was clearly generated by reflex pathways, presumably arising from mechanoreceptors on the gills, because the spontaneous breathing efforts of the intubated fish were out of phase with imposed changes in water velocity and were without effect on heart beat.

Recent work on pacu, Piaractus mesopotamicus $(28,39)$, has revealed that respiration-related, bursting activity is only recorded from the cardiac vagus when fish are hyperventilating or coughing, implying that the bursts arise reflexly, following stimulation of branchial mechanoreceptors. The spontaneous occurrence of a cough or bout of increased ventilatory effort was accompanied by bursts of activity in the cardiac vagus, with consequent transient bradycardia. In fish rendered moderately hypoxic by reduction of the flow of water irrigating the gills, a period of spontaneously increased ventilatory amplitude was accompanied by respiration-related bursts of activity in the cardiac vagus, which were not apparent in the inactive normoxic fish and appeared to recruit the heart. As in the dogfish, phasic peripheral stimulation of the cardiac vagus or central stimulation of respiratory branches of cranial nerves VII, IX and X entrained the heart over a wide range of frequencies, both below and above the intrinsic rate $(28,39)$. Thus increased ventilatory efforts generate activity in the cardiac vagus, slowing or recruiting the heart.

Thus, we are left with an apparent conflict of evidence on the mode of generation of cardiorespiratory synchrony, which in elasmobranches may be primarily generated by central interactions in inactive, normoxic, or hyperoxic fish when cardiac vagal tone is low; while in teleosts it appears during hypoxia perhaps as a result of stimulation of branchial mechanoreceptors during forced ventilation and is generated reflexly by increased vagal tone. There is, however, some evidence from our current studies that central interactions may to some extent generate cardiorespiratory synchrony in teleosts. Recordings of respiration-related bursting activity in the cardiac nerve of pacu was found to be concurrent with bursts in the mandibular branch of the Vth cranial nerve and both anticipated activity in the respiratory branches of the VIIth, IXth, and Xth cranial nerves (Figure 2A) (39). This was also the case in recordings from dogfish. In both elasmobranches and teleosts the distribution of motor neurons supplying the $\mathrm{V}$ th nerve in the brainstem does not overlap with the distribution of CVPN or with neurons supplying the other respiratory nerves (Figure $2 B)(1,21,39)$. In addition, central electrical stimulation of the mandibular branch of the Vth cranial nerve in pacu was without effect on heart rate, while central stimulation of respiratory branches of the VIIth, IXth and Xth cranial nerves either slowed or recruited the heart (21). Accordingly, concurrence of bursting activity in the cardiac nerve with activity in the Vth cranial nerve is likely to be generated centrally, possibly by inputs to both groups of neurons from the central respiratory pattern generator when respiratory drive is high, as in hypoxia. This possibility merits further investigation with the dogfish as the model of choice.

\section{Air-breathing fish}

Many of the primitive ray-finned fishes are air-breathers and air-breathing also seems to have evolved numerous times within teleosts (40). The anatomy and physiology of the air-breathing organs vary enormously among species, but it is characteristic that heart rate increases during airbreathing. In lungfish (Dipnoi), a vagal innervation of the heart is well established, whilst the sympathetic chain seems poorly developed (41). Heart rate does not change much with air breathing but pulmonary ventilation is associated with a marked rise in pulmonary blood flow in all species of lungfish (for a review, see Ref. 42). Control of pulmonary blood flow involves branchial shunts, which are neurally regulated and cholinergic vasoconstriction of the 
pulmonary artery. Lungfish are endowed with pulmonary stretch receptors as well as both central and peripheral chemoreceptors, making it likely that feedback from these receptors is involved.

Changes in heart rate during ventilation are also evident in the jeju, Hoplerythrinus unitaeniatus, a teleost fish that also uses a modified swim bladder as an air-breathing organ. Heart rate decreases during expiration, but increases drastically during the subsequent inhalation that inflates the swim bladder (43). These heart rate changes were primarily of cholinergic origin and an in-depth analysis of HRV in this species indicated that the heart rate changes were qualitatively similar to RSA in higher vertebrates (43). An example of the rapid change in heart rate upon an airbreath in Synbranchus marmoratus is shown in Figure 3

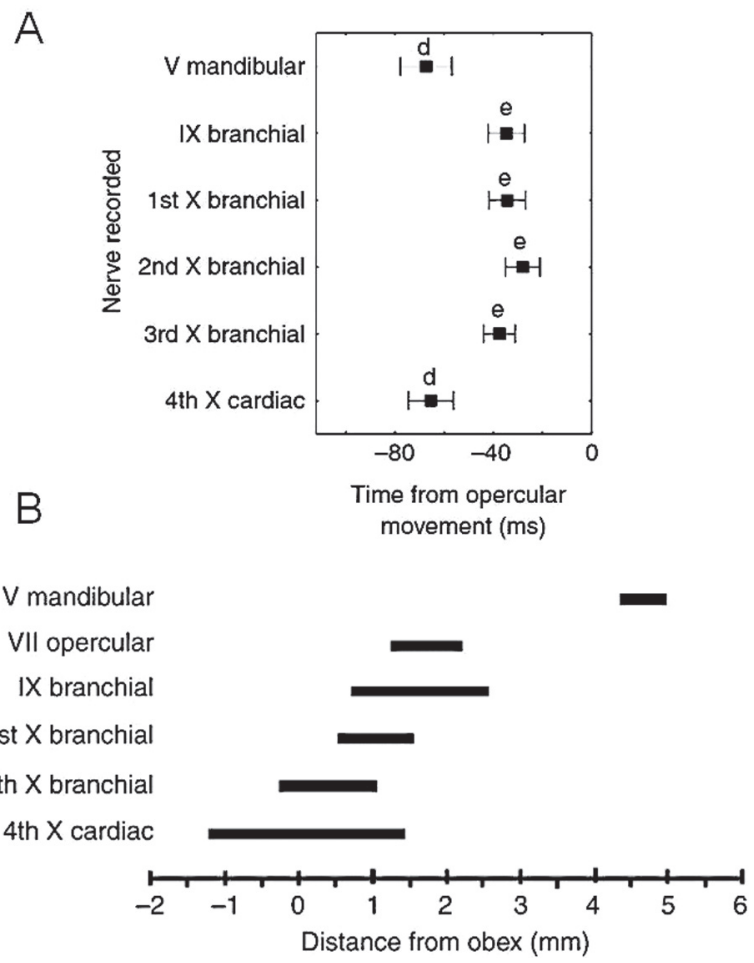

Figure 2. $A$, Mean duration ( $\pm \mathrm{SD}$ ) of the interval between each peak (filled squares) of the burst activity in respiratory branches of cranial nerves $\mathrm{V}, \mathrm{IX}$, and $\mathrm{X}$ or the cardiac branch of $\mathrm{X}$ and the peak of the succeeding opercular movement (time $=0$ ) in Piaractus mesopotamicus. The letters $\mathrm{d}$ and e denote significantly similar groups of mean values. The peaks of activity in the cardiac nerve are concurrent with activity in the Vth cranial nerve and both anticipate activity in the IXth and 1st, 2nd and 3rd respiratory branches of the Xth cranial nerve. $B$, The rostrocaudal distribution in the brainstem of $P$. mesopotamicus of cell bodies of respiratory visceral motor neurons supplying axons to the mandibular Vth, opercular VIIth, branchial IXth, and 1st and 4th branchial $X$ th, together with cardiac vagal preganglionic neurons supplying axons to the cardiac vagus (modified from Ref. 12, with permission). (from Ref. 44). As seen in this example, expansion of the buccopharyngeal cavity leads to a marked rise in heart rate (44).

\section{Amphibians}

There are limited data on the central topography of the vagal motor column in amphibians. In the African clawed toad Xenopus laevis, about $30 \%$ of VPN are located in a ventrolateral location outside the DVN. Both groups innervate all target organs including the heart and lungs (45). In the neotenous axolotl, Ambystoma mexicanum that retains larval features into the adult (i.e., sexually mature) stage, including external gills and gill clefts in the pharynx, all VPN are in a medial nucleus representing the DVN. When induced to metamorphose, they lose their gills and leave water to become committed lung breathers. There is an increase in the number of VPN and a proportion of them (about 15\%) are found in a more lateral location in the white matter of the medulla (46). This relocation may in part relate to the switch from gill to lung-breathing.

An authoritative review that considered the influences of phylogeny, ontogeny and season on central cardiovascular function in amphibians has reported that early-stage fully aquatic larvae of the bullfrog show no evidence of reflex adjustments of heart rate (47). Cholinergic sensitivity of the cardiac pacemaker increases during larval development and a vagal tone on the heart is first apparent at the onset of air-breathing. At metamorphosis there is a sharp decrease in cholinergic sensitivity and adult bullfrogs show

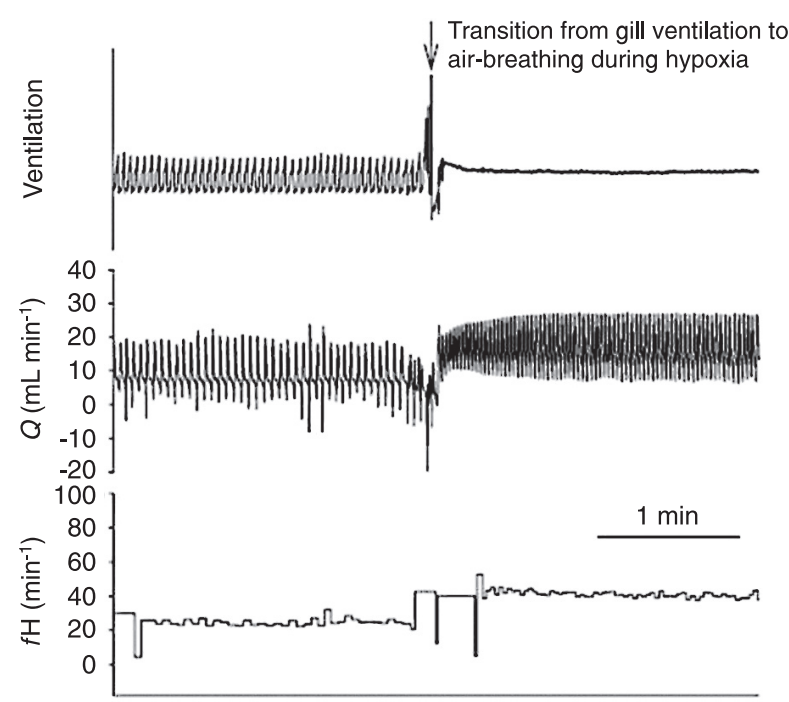

Figure 3. Changes in the ventilatory and cardiac parameters during transition from gill ventilation to air-breathing in Synbranchus marmoratus in hypoxic water $\left(\mathrm{PO}_{2} 50 \mathrm{mmHg}\right)$. The arrow indicates the air-breathing time. $\mathrm{Q}=$ cardiac output; $f_{\mathrm{H}}=$ heart rate (modified from Ref. 44, with permission). 
no resting vagal or adrenergic tone on the heart. However, cardiac vagal tone varies widely with temperature in some adult amphibians. In Xenopus, cardiac vagal tone increased markedly with temperature between $10^{\circ}$ and $30^{\circ} \mathrm{C}$, causing a major modulation of the Q10 relationship for heart rate. However, the situation was complicated by the presence of a ß-adrenergic tone on the heart that also varied with temperature. Injection of propranolol plus atropine revealed the true level of the predominant vagal tone (1). Altimiras et al. (48) studied these interactions between the two arms of autonomic control of the heart in teleost fishes and showed that the order in which antagonists are injected changes the calculated values for sympathetic and parasympathetic tone.

Amphibians have evolved control mechanisms, which relate to whether they are more or less committed to lung breathing. Those that are less committed to air-breathing or have no lungs, like the lungless salamanders, Plethodontidae, rely solely on sympathetic adrenergic regulation of cutaneous blood flow to control blood gases (49). In intermittent lung breathers like the frog and toad, control of pulmonary blood flow is achieved by a strong vagal cholinergic vasoconstriction of the pulmo-cutaneous artery that is extrinsic to the lung (50). Vasoconstriction in the pulmonary circuit reduces pulmonary blood flow and increases systemic recirculation of oxygen-poor blood from the right atrium, whereas decreased vagal tone on the pulmonary artery is associated with the increased pulmonary blood flow observed during lung ventilation. Adrenergic sympathetic vasoconstriction of the cutaneous circulation contributes secondarily to increases in pulmonary blood flow (50). The extent to which withdrawal of vagal tone versus increased sympathetic tone contributes to the increased heart rate and pulmonary blood flows associated with lung ventilation is not resolved but it may be primarily due to release of vagal tone, as vagotomy or injection of atropine reduces or abolishes cardiorespiratory coupling (45).

Although most anuran larvae show unchanging heart rates during episodic lung ventilation (47), there is a clear cardiorespiratory coupling in adult anurans, in that intermittent lung ventilation is matched by intermittent increases in pulmonary blood flow, without compromising systemic blood flow. The mechanisms underlying these relationships are unknown. Recent experiments demonstrated increases in heart rate and pulmonary blood flow during bouts of fictive breathing in decerebrated paralyzed and through ventilated toads, indicating central control of cardiorespiratory interactions (45). These may in part arise from the overlapping central topography of CVPN and pulmonary VPN $(45,46)$. Alternatively, stimulation of lung stretch receptors during bouts of breathing may result in release of vagal tone on the heart and pulmonary artery. Artificial inflation of the lungs in anesthetized frogs and toads elicited cardiovascular responses similar to those observed in normally breathing animals, which were abolished by deep anesthesia or injec- tion of atropine (45). However, in conscious Xenopus, denervation of pulmonary stretch receptors did not abolish the increase in heart rate associated with lung inflation (1).

Some of the cardiac responses to intermittent lung ventilation may be generated directly by mechanical or chemical factors (45). In anesthetized toads artificial lung inflation caused increased pressure in the left atrium and an elevated heart rate, which was not abolished by atropine injection, implying that direct mechanical effects on venous return to the heart (i.e., the Frank-Starling mechanism) may contribute to cardiorespiratory coupling. An alternative mechanism has been proposed for anesthetized and unidirectionally ventilated toads, in which hypoxia and hypercapnia reduced pulmonary blood flow. Some of this response may be locally mediated by a direct effect on vascular tone.

\section{Reptiles}

Reptiles are typically periodic breathers, and during bouts of breathing the degree of shunting of blood flow to the lung increases. Vasomotor control is important in diverting blood between the pulmonary and systemic systems. In turtles and lizards the net direction and magnitude of shunt flow is affected by resistance in the pulmonary circuit, relative to the systemic circuit, by active vagal, cholinergic regulation of pulmonary arterial resistance. Control of pulmonary blood flow in reptiles is achieved by vagal cholinergic constriction of the pulmonary artery. Peripheral electrical stimulation of the vagus or intravenous injection of acetylcholine results both in bradycardia and an increase in pulmonary vascular resistance, which reduces pulmonary blood flow (1). These cardiovascular changes are abolished by administration of atropine. The rattlesnake, Crotalus durissus, has a single lung and functional pulmonary arch, designated as the right arch, though it is innervated by the left vagus. Peripheral electrical stimulation of the left vagus slowed the heart and stopped blood flow to the pulmonary arch (51). Blood flow is also under adrenergic control. Vagal cholinergic tone predominated over sympathetic adrenergic tone in inactive Boa constrictor. The increase in heart rate during enforced activity was due largely to complete withdrawal of inhibitory vagal tone, while the increase following a meal was mediated by small changes in overall autonomic tone with evidence of involvement of non-adrenergic-non-cholinergic (NANC) factors $(23,24)$.

Reptiles show clear examples of cardiorespiratory coupling. In the free diving turtle, Trachemys scripta, pulmonary blood flow increased more than 3-fold at the onset of breathing, during recovery from breath-holds lasting longer than $5 \mathrm{~min}$ (52). Systemic blood flow also increased during ventilation. These increases were accomplished entirely through changes in heart rate during ventilation, with stroke volume unchanged. Systemic blood flow always exceeded pulmonary flow so that a net right to left cardiac 
shunt prevailed, regardless of ventilatory state. Nevertheless, because pulmonary flow increased markedly during ventilation, the ratio of pulmonary to systemic flow increased from 0.3 to 0.8 . These cardiovascular changes associated with intermittent lung ventilation in discontinuous breathers have been referred to as cardiorespiratory synchrony (e.g., 52), which is a different use of the term compared to one-to-one synchrony in fish (see above).

In both, the turtle, Pseudemys scripta, and the tortoise, Testudo graeca, the onset of lung ventilation was closely accompanied by a tachycardia (53). As stimulation of pulmonary stretch receptors, arterial chemoreceptors and baroreceptors or water receptors was without effect on heart rate, it was concluded that this ventilatory tachycardia resulted from central interactions between respiratory and cardiac neurons in the medulla. As the breathing tachycardia was unaffected by $\beta$-adrenergic blockade, it seems that all changes in heart rate were mediated by alterations in vagal tone. Heart rate fell slightly before and markedly after hatching in the snapping turtle, Chelydra serpentina, indicating the establishment of a vagal tone on the heart, coincident with the onset of lung breathing (54). The heart rate changes with ventilation disappear upon blockade of the autonomic nervous innervation of the heart and are clearly regulated.

Wang et al. (23) showed that there are slight changes in $f_{\mathrm{H}}$ related to lung ventilation in snakes but it is uncertain if these components formed distinct oscillations in $f_{\mathrm{H}}$ at the frequency of $f_{R}$, and therefore could be categorized as RSA. On the basis of power spectral analysis of heart rate, there was no spectral component of the heart rate

A

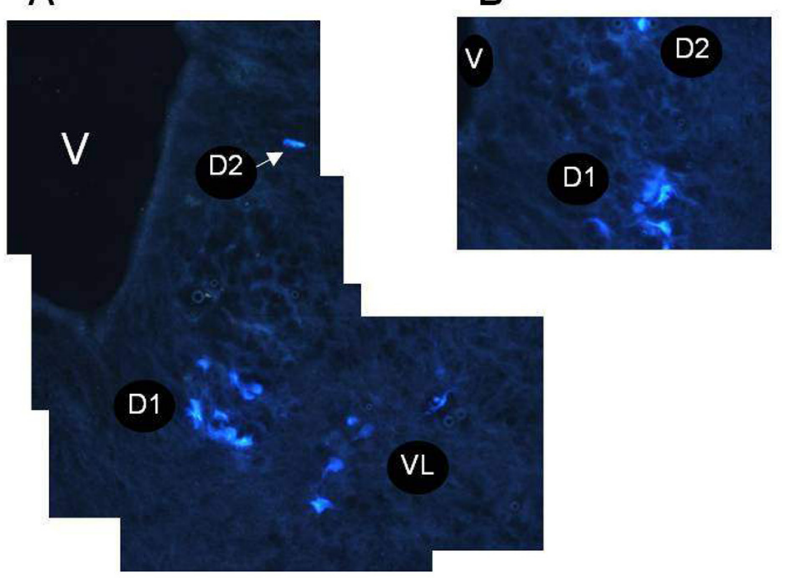

Figure 4. Transverse sections of the caiman brainstem (A) 1.02 $\mathrm{mm}$ and (B) $0.42 \mathrm{~mm}$ rostral to the obex. The cell bodies of the vagal preganglionic neurons were identified by injection of the neural tracer True Blue into the cervical vagus. Cell bodies were located in two distinct groups in the dorsal vagal motor nucleus (DVN) (D1 and D2) close to the edge of the 4th ventricle (V) and in a ventrolateral group (VL) outside the DVN. signal with ventilation in a small lizard Galloti galloti (55). However, the use of $f_{\mathrm{H}}$ dataloggers for long-term monitoring in undisturbed rattlesnakes (22) enabled us to determine HRV in settled, recovered animals when sympathetic tonus was low and vagal tonus high. These animals showed oscillatory components in the HRV signal at the frequency of ventilation that were abolished by injection of atropine. Results from this study agreed in part with Gonzalez and De Vera (55), in that two peaks were detected in the $f_{\mathrm{H}}$ spectra of the rattlesnake. However, the frequency and amplitude of these peaks was relative to $f_{\mathrm{H}}$, with high $f_{\mathrm{H}}$ favoring the lower frequency peaks and low $f_{\mathrm{H}}$ the high frequency peaks. The high frequency peaks that were removed by the cholinergic blocker atropine occurred at the frequency of the respiratory cycle. The respiratory cycle of rattlesnakes consists of a prolonged inspiration followed by a relatively short expiration. Heart rate slowed upon expiration and increased during inspiration, which is similar to the changes in heart rate observed in conscious unrestrained mammals and characterized as RSA (16). Thus, this study contrasts with that of Gonzalez and De Vera (55), as we were able to present clear evidence for respiratory modulation of heart rate that closely resembled that recorded from mammals and accordingly may be classed as RSA.

Accordingly, the previous data refute the proposition that centrally controlled cardiorespiratory coupling is restricted to mammals, as propounded by the polyvagal theory of Porges (19). Neural tracers applied to the vagus nerve in the caiman revealed VPN both within and outside the DVN (Figure 4), with about $15 \%$ in the ventrolateral group that may constitute a primitive NA (Figure $5 \mathrm{~B}$ ). In an associated neuroanatomical study we were able to show that VPN were distributed in 3 locations in the brainstem of the rattlesnake, 2 in the DVN and a ventrolateral group outside of the DVN (Figure 5A) (22). So the mammalian-style RSA in reptiles may have its basis in a dual location for CVPN as described in mammals where RSA is generated largely in the NA (1).

\section{Non-adrenergic-non-cholinergic control of heart rate}

An increase in heart rate after a meal (postprandial tachycardia) has been reported for most groups of vertebrates, including reptiles (see, for instance, Refs. 23,24) and humans. The underlying regulatory mechanism remains elusive. Interestingly, however, patients with transplanted and fully denervated hearts exhibit a pronounced postprandial rise in cardiac output (56), suggesting that the cardiac stimulation is not reflex in nature, but, at least partially mediated by humoral factor(s).

In boas and pythons, digestion causes a pronounced and long-lasting increase in heart rate $(23,57)$. The postprandial tachycardia is proportional to meal size and in pythons digesting a meal amounting to $25 \%$ of the snakes 

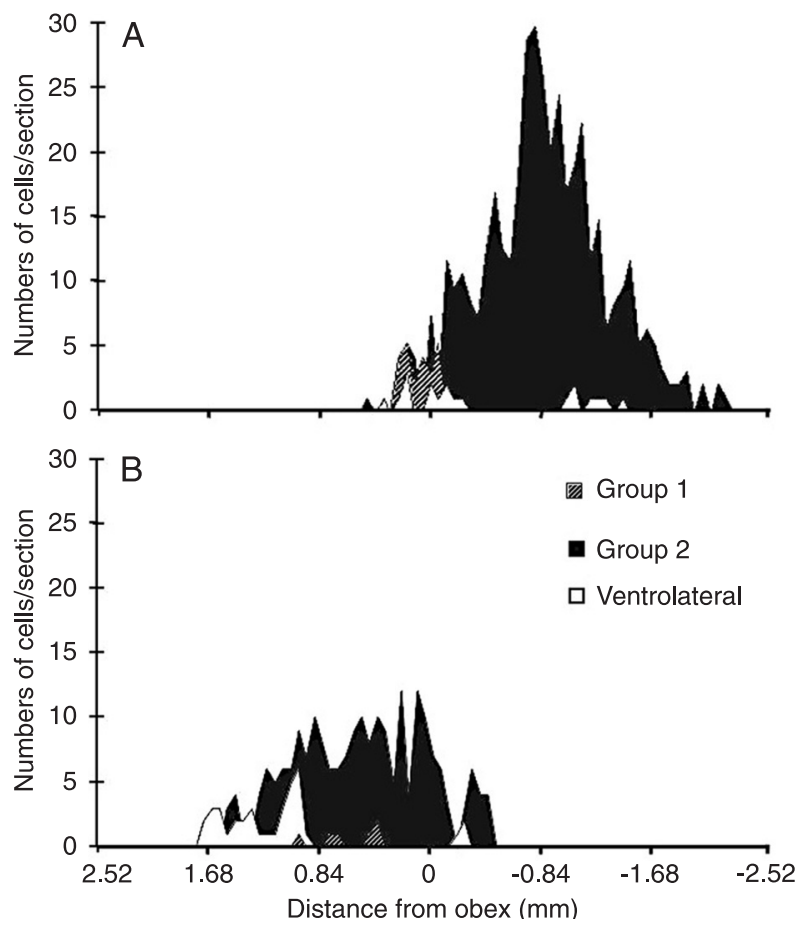

Figure 5. Numbers of cell bodies of vagal preganglionic neurons (VPN) plotted against their rostrocaudal distribution around the obex in the brainstem of two reptiles, $(A)$ rattlesnake and $(B)$ caiman. The black areas denote the distribution of the major group of VPN in the dorsal vagal motor nucleus (DVN), the grey area denotes a smaller group of cells in the DVN and the white areas denote the scattered distribution of VPN ventrolaterally, outside the DVN. The two groups of VPN in the DVN have overlapping distributions rostral to the obex. There are a small number of cell bodies (white) located laterally outside of the DVN, that comprise about $4 \%$ of the total in the rattlesnake and $15 \%$ of the total in the caiman (the rattlesnake data are modified from Ref. 13, with permission, and the caiman data are unpublished).

body weight heart rate doubled $24 \mathrm{~h}$ into digestion. The postprandial tachycardia is mediated by a withdrawal of the inhibitory vagal tone and an increase in intrinsic heart rate revealed after pharmacological blockade of both muscarinic and $\beta$-adrenergic receptors $(23,57)$. This implies that other factors than catecholamines or reduced cholinergic tone exert a pronounced positive chronotropic action during digestion. This NANC stimulation of the heart could arise from increased circulating levels of a hormone that either acts directly on the heart or presynaptically on

\section{References}

1. Taylor EW, Jordan D, Coote JH. Central control of the cardiovascular and respiratory systems and their interactions in vertebrates. Physiol Rev 1999; 79: 855-916.

2. Cechetto D, Gelb AW. The amygdala and cardiovascular cardiac neurons causing release of chronotropic agents. In humans, postprandial heart rate is also stimulated by an NANC factor (58). In digesting sea bass, however, the increase in heart rate is caused only by a withdrawal of cholinergic tone.

Interestingly, histamine exerted a positive chronotropic effect on intrinsic heart rate in fasting pythons, which was abolished in digesting snakes (57). Furthermore, injection of the histamine $\mathrm{H}_{2}$-receptor antagonist ranitidine decreased intrinsic heart rate by about 20 beats/min in digesting snakes but had no effect in fasting snakes. This suggests that there is no histaminergic tone on the heart in fasting snakes, but that a large histaminergic tone develops by $24 \mathrm{~h}$ into the postprandial period. The histaminergic tone on the heart disappears by $48 \mathrm{~h}$ into the postprandial period while the intrinsic heart rate is still elevated. Thus, while increased histaminergic tone appears to explain most of the tachycardia during the initial phase of digestion, other NANC factors seem to be involved in regulating the postprandial heart rate, in particular during the subsequent phases of digestion. It is tempting to speculate that, as the ingested food is being processed and moves along in the gastrointestinal system, this progressively stimulates the release of various hormones and regulatory peptides, which then directly or indirectly induce the responses to digestion including the postprandial increase in cardiac output and heart rate. Other hormones released from the duodenal-pancreatic region have been suggested to be involved in the regulation of the postprandial increase in cardiac output and heart rate. Insulin has been considered because of its cardiovascular effects including a positive chronotropic and ionotropic effect. Secretin increases heart rate and cardiac output during digestion in dogs and humans (59) and the intestinal hormone oxyntomodulin, which is released during digestion, increases intrinsic heart rate in mice when administered peripherally (60).

\section{Acknowledgments}

E.W. Taylor was a visiting Professor at UFSCar and UNESP in 2006, supported by FAPESP. C.A.C. Leite is a postdoctoral researcher at UNESP supported by FAPESP. N. Skovgaard was a Postdoctoral researcher in the University of Notre Dame, IN, USA, supported by the Danish Research Council and the Villum Kann Rasmussen Foundation. E.W. Taylor and C.A.C. Leite are collaborators on the INCT em Fisiologia Comparada supported by CNPq and FAPESP. control. J Neurosurg Anesthesiol 2001; 13: 285-287.

3. Cechetto DF. Forebrain control of healthy and diseased hearts. In: Armour JA, Ardell JL (Editors), Basic and clinical neurocardiology. Oxford: University Press; 2004. p 220- 
251.

4. Hainsworth R. Reflexes from the heart. Physiol Rev 1991; 71: 617-658.

5. Daly MB. Peripheral arterial chemoreceptors and respiratory-cardiovascular integration. Oxford: Oxford University Press; 1997.

6. Marshall JM. Peripheral chemoreceptors and cardiovascular regulation. Physiol Rev 1994; 74: 543-594.

7. Butler PJ, Jones DR. Physiology of diving of birds and mammals. Physiol Rev 1997; 77: 837-899.

8. van Giersbergen PL, Palkovits M, De Jong W. Involvement of neurotransmitters in the nucleus tractus solitarii in cardiovascular regulation. Physiol Rev 1992; 72: 789-824.

9. Otake K, Ezure K, Lipski J, Wong She RB. Projections from the commissural subnucleus of the nucleus of the solitary tract: an anterograde tracing study in the cat. J Comp Neurol 1992; 324: 365-378.

10. Finger TE, Kanwal JS. Ascending general visceral pathways within the brainstems of two teleost fishes: Ictalurus punctatus and Carassius auratus. J Comp Neurol 1992; 320: 509-520.

11. Meek J, Nieuwenhuys R. Holosteans and teleosts. In: Nieuwenhuys HI, Donkelaar T, Nicholson C (Editors), The central nervous system of vertebrates. Vol. 2. Berlin: Springer; 1998. p 759-937.

12. Sundin L, Turesson J, Taylor EW. Evidence for glutamatergic mechanisms in the vagal sensory pathway initiating cardiorespiratory reflexes in the shorthorn sculpin Myoxocephalus scorpius. J Exp Biol 2003; 206: 867-876.

13. Daly MB, Kirkman E. Differential modulation by pulmonary stretch afferents of some reflex cardioinhibitory responses in the cat. J Physiol 1989; 417: 323-341.

14. Jones JFX, Wang Y, Jordan D. Activity of cardiac vagal preganglionic neurones during the pulmonary chemoreflex in the anaesthetized cat. In: O'Regan RG, McQueen DS, Paterson DJ (Editors), Chemoreceptors and chemoreceptor reflexes in health and disease. New York: Plenum Press; 1994. p 301-303.

15. Jordan D, Spyer KM. Central neural mechanisms mediating respiratory-cardiovascular interactions. In: Taylor EW (Editor), Neurobiology of the cardiorespiratory system. Manchester: Manchester University Press; 1987. p 322-341.

16. Hayano J, Yasuma F. Hypothesis: respiratory sinus arrhythmia is an intrinsic resting function of cardiopulmonary system. Cardiovasc Res 2003; 58: 1-9.

17. Windle WF. Neurofibrillar development in the central nervous system of cat embryos between 8 and $12 \mathrm{~mm}$ long. J Comp Neurol 1933; 58: 643-723.

18. Thompson CR, Brown JS, Gee H, Taylor EW. Heart rate variability in healthy term newborns: the contribution of respiratory sinus arrhythmia. Early Hum Dev 1993; 31: 217-228.

19. Porges SW. Orienting in a defensive world: mammalian modifications of our evolutionary heritage. A polyvagal theory. Psychophysiology 1995; 32: 301-318.

20. Grossman P, Taylor EW. Toward understanding respiratory sinus arrhythmia: relations to cardiac vagal tone, evolution and biobehavioral functions. Biol Psychol 2007; 74: 263285.

21. Taylor EW. Nervous control of the heart and cardiorespiratory interactions. In: Hoar WS, Randall DJ, Farrell AP (Editors), Fish physiology. Vol. XIIB. New York: Academic Press; 1992. p 343-387.
22. Campbell HA, Leite CA, Wang T, Skals M, Abe AS, Egginton $S$, et al. Evidence for a respiratory component, similar to mammalian respiratory sinus arrhythmia, in the heart rate variability signal from the rattlesnake, Crotalus durissus terrificus. J Exp Biol 2006; 209: 2628-2636.

23. Wang T, Taylor EW, Andrade D, Abe AS. Autonomic control of heart rate during forced activity and digestion in the snake Boa constrictor. J Exp Biol 2001; 204: 3553-3560.

24. Wang T, Warburton S, Abe A, Taylor T. Vagal control of heart rate and cardiac shunts in reptiles: relation to metabolic state. Exp Physiol 2001; 86: 777-784.

25. Farrell AP, Sobin SS, Randall DJ, Crosby S. Intralamellar blood flow patterns in fish gills. Am J Physiol 1980; 239: R428-R436.

26. Randall DJ. Nervous control of cardiac activity in tench (Tinca tinca) and goldfish (Carassius auratus). Physiol Zool 1966; 39: 185-192.

27. Leite CA, Florindo LH, Kalinin AL, Milsom WK, Rantin FT. Gill chemoreceptors and cardio-respiratory reflexes in the neotropical teleost pacu, Piaractus mesopotamicus. J Comp Physiol A Neuroethol Sens Neural Behav Physiol 2007; 193: 1001-1011.

28. Leite CA, Taylor EW, Guerra CD, Florindo LH, Belao T, Rantin FT. The role of the vagus nerve in the generation of cardiorespiratory interactions in a neotropical fish, the pacu, Piaractus mesopotamicus. J Comp Physiol A Neuroethol Sens Neural Behav Physiol 2009; 195: 721-731.

29. Campbell HA, Taylor EW, Egginton S. The use of power spectral analysis to determine cardiorespiratory control in the short-horned sculpin Myoxocephalus scorpius. J Exp Biol 2004; 207: 1969-1976.

30. Taylor EW, Butler PJ. Nervous control of heart rate: activity in the cardiac vagus of the dogfish. J Appl Physiol 1982; 53: 1330-1335.

31. Taylor EW, Leite CA, Levings JJ. Central control of cardiorespiratory interactions in fish. Acta Histochem 2009; 111: 257-267.

32. Barrett DJ, Taylor EW. The characteristics of cardiac vagal preganglionic motoneurones in the dogfish. J Exp Biol 1985; 117: 459-470.

33. De Burgh Daly M, Scott MJ. An analysis of the primary cardiovascular reflex effects of stimulation of the carotid body chemoreceptors in the dog. J Physiol 1962; 162: 555-573.

34. Taylor EW, Campbell HA, Levings JJ, Young MJ, Butler PJ, Egginton $S$. Coupling of the respiratory rhythm in fish with activity in hypobranchial nerves and with heartbeat. Physiol Biochem Zool 2006; 79: 1000-1009.

35. Levy MN, Martin PJ, Stuesse SL. Neural regulation of the heart beat. Annu Rev Physiol 1981; 43: 443-453.

36. Brown GL, Eccles JC. The action of a single vagal volley on the rhythm of the heart beat. J Physiol 1934; 82: 211-241.

37. Pace DG, Masuda Y, Eisenstein I, Levy MN. Effects of digoxin on the chronotropic responses to repetitive vagal stimulus bursts in the dog. Can J Physiol Pharmacol 1984; 62: 1411-1415.

38. Randall DJ, Smith JC. The regulation of cardiac activity in fish in a hypoxia environment. Physiol Zool 1967; 40: 104113.

39. Taylor EW, Leite CA, Florindo LH, Belao T, Rantin FT. The basis of vagal efferent control of heart rate in a neotropical fish, the pacu, Piaractus mesopotamicus. J Exp Biol 2009; 212: $906-913$. 
40. Graham JB. Air breathing fishes: evolution, diversity, and adaptation. San Diego: Academic Press; 1997.

41. Axelsson M, Abe AS, Bicudo JEPW, Nilsson S. On the cardiac control in the South-American lungfish, LepidosirenParadoxa. Comp Biochem Physiol A Physiol 1989; 93: 561. 565.

42. Taylor EW, Wang T. Control of the heart and of cardiorespiratory interactions in ectothermic vertebrates. In: Glass ML, Wood SC (Editors), Cardio-respiratory control in vertebrates: Comparative and evolutionary aspects. New York: Springer; 2009. p 285-316.

43. McKenzie DJ, Campbell HA, Taylor EW, Micheli M, Rantin FT, Abe AS. The autonomic control and functional significance of the changes in heart rate associated with air breathing in the jeju, Hoplerythrinus unitaeniatus. J Exp Biol 2007; 210: 4224-4232.

44. Skals M, Skovgaard N, Taylor EW, Leite CA, Abe AS, Wang T. Cardiovascular changes under normoxic and hypoxic conditions in the air-breathing teleost Synbranchus marmoratus: importance of the venous system. J Exp Biol 2006; 209: 4167-4173.

45. Wang T, Hedrick MS, Inmied YM, Taylor EW. Control and interaction of the cardiovascular and respiratory systems in anuran amphibians. Comp Biochem Physiol A Mol Integr Physiol 1999; 124: 393-406.

46. Taylor EW, Al-Ghamdi MS, Ihmied IH, Wang T, Abe AS. The neuranatomical basis of central control of cardiorespiratory interactions in vertebrates. Exp Physiol 2001; 86: 771-776.

47. Burggren WW. Central cardiovascular function in amphibians: qualitative influences of phylogeny, ontogeny and seasonality. In: Heisler N (Editor), Mechanisms of systemic regulation. Respiration and circulation. Vol. 1. Berlin: Springer-Verlag; 1995. p 175.

48. Altimiras J, Aissaoui A, Tort L, Axelsson M. Cholinergic and adrenergic tones in the control of heart rate in teleosts. How should they be calculated? Comp Biochem Physiol 1997; 118A: 131-139.

49. Farrell AP. From hagfish to tuna: a perspective on cardiac function. Physiol Zool 1997; 64: 1137-1164.

50. West $\mathrm{NH}$, Burggren WW. Factors influencing pulmonary and cutaneous arterial blood flow in the toad, Bufo marinus. Am J Physiol 1984; 247: R884-R894.

51. Taylor EW, Andrade DV, Abe AS, Leite CA, Wang T. The unequal influences of the left and right vagi on the control of the heart and pulmonary artery in the rattlesnake, Crotalus durissus. J Exp Biol 2009; 212: 145-151.

52. Wang T, Hicks JW. Cardiorespiratory synchrony in turtles. $J$ Exp Biol 1996; 199: 1791-1800.

53. Burggren WW. A quantitative analysis of ventilation tachycardia and its control in two chelonians, Pseudemys scripta and Testudo graeca. J Exp Biol 1975; 63: 367-380.

54. Birchard GF, Reiber CL. Heart rate during development in the turtle embryo: effect of temperature. J Comp Physiol B 1996; 166: 461-466.

55. Gonzalez Gonzalez J, De Vera Porcell L. Spectral analysis of heart rate variability of lizard, Gallotia galloti. Am J Physiol 1988; 254: R242-R248.

56. Waaler BA, Hisdal J, Ihlen H, Kjekshus J. Mechanisms behind the postprandial increase in cardiac output: a clue obtained from transplanted hearts. Eur J Appl Physiol 2006; 97: $516-520$

57. Skovgaard N, Moller K, Gesser H, Wang T. Histamine induces postprandial tachycardia through a direct effect on cardiac H2-receptors in pythons. Am J Physiol Regul Integr Comp Physiol 2009; 296: R774-R785.

58. Kelbaek H, Munck O, Christensen NJ, Godtfredsen J. Autonomic nervous control of postprandial hemodynamic changes at rest and upright exercise. J Appl Physiol 1987; 63: 1862-1865.

59. Gunnes P, Smiseth OA, Lygren I, Jorde R. Effects of secretin infusion on myocardial performance and metabolism in the dog. J Cardiovasc Pharmacol 1985; 7: 1183-1187.

60. Sowden GL, Drucker DJ, Weinshenker D, Swoap SJ. Oxyntomodulin increases intrinsic heart rate in mice independent of the glucagon-like peptide-1 receptor. Am J Physiol Regul Integr Comp Physiol 2007; 292: R962-R970. 